\title{
The influence of punch blunting on the elastic indentation response
}

\author{
A M Korsunsky \\ Department of Engineering Science, University of Oxford, Parks Road, Oxford OX1 3PJ, UK
}

\begin{abstract}
Elastic solutions of a family of axisymmetric problems concerning frictionless contact between a rigid punch and a semi-infinite substrate are considered using the method of Green and Zerna and of Collins. The analysis is relevant to the interpretation of experimental results in materials indentation testing, e.g. when substrate properties need to be determined from load-displacement traces, and precise information about the indenter tip shape is crucial.

Commonly used solutions for ideal punch shapes, e.g. those having spherical or conical tips, may only be viewed as approximations since, in practice, indenter tips are neither perfectly round nor infinitely sharp. In order to illustrate the influence that small variations in punch shape may have on the contact behaviour, analytical solutions for a blunted Hertzian indenter and a rounded cone are obtained in parametric form, and their asymptotic behaviour at the extremes of low and high loads is investigated. A smooth punch is then considered of a general shape, given by a power series, and the resulting general solution is used as a basis for developing an inverse problem formulation of the tip shape calibration procedure. The method allows the best match between the measured and predicted load-displacement dependencies to be established. An example of the application of this procedure to the analysis of some nanoindentation data is presented.
\end{abstract}

Keywords: indentation, frictionless elastic contact, materials characterization

\section{INTRODUCTION}

Materials testing using indentation is an appealingly simple technique. In its most straightforward and traditional form it involves making an impression in the surface of a specimen with a small hard indenter of known geometry under the application of a carefully controlled load. The ratio of this load to the area of the permanent impression remaining after the indenter is removed is thought to be a material property. It is known as hardness and relates to the yield strength and the hardening behaviour of the specimen material.

Over the last few decades, some important developments have been introduced to this technique.

Firstly, progressively smaller loads (and, therefore, impression sizes) were used. The reasoning behind this shift towards microhardness testing was that it allows the material response to be interrogated at progressively smaller scales, thus revealing information about local microstructural features and properties, instead of reflecting the average macroscopic behaviour alone, as in conventional hardness testing.

The MS was received on 16 November 2000 and was accepted after revision for publication on 3 April 2001.
Secondly, the indentation instrumentation progressively acquired further electronic attachments, carrying out control, monitoring and data collection tasks. These developments produced equipment capable of continuously recording the variations in load, tip displacement, speed and other parameters in the experiment. In this way, vast amounts of data could be collected which undoubtedly contain much useful identifying information about material behaviour. For this reason the technique came to be called 'materials fingerprinting' (somewhat inappropriately, since the diamond indenter tips are very stiff and hard and undergo only very small elastic deformation; it is the indenter that leaves an impression in the specimen's surface and not vice versa).

Against the background of these remarkable advances in experimental methods, the interpretation and modelling approaches used in order to analyse the test results largely remained confined to extracting the same information as in conventional tests, i.e. the hardness and contact compliance. In terms of the modelling methodology, either classical elastic indentation solutions were employed, or specific finite element models were developed.

An important issue in all very low-load experiments also concerns the assumptions made about the contact geometry. At the scales considered, no indenter ever exhibits the perfect self-similarity required of the pyramidal tips (four- 
sided Vickers or three-sided Berkovich). Also, even the best attempts at preparing a perfectly rounded diamond tip for simulating Hertzian contact inevitably produce flattened imperfect shapes.

The situation is illustrated in Fig. 1, which shows a surface map of a $500 \mathrm{mN}$ indentation made in fused silica using a Nanoindenter II instrument with a nominally Hertzian tip of radius $25 \mu \mathrm{m}$. The image was recorded using a Park atomic force microscope in contact mode. Deviations from perfect axial symmetry are evident, with the indentation shape resembling a blunted three-sided pyramid. This situation could be also described as variations in the curvatures found in the cross-sections made through the centre of impression at different azimuthal angles. If an equivalent axisymmetric shape were to be chosen to describe the indenter shape, it is likely to differ significantly from the perfect Hertzian (parabolic) shape.

The above considerations formed the motivation for the analysis of elastic contact problems for rigid punches of some special and general axisymmetric shapes, which is presented in this paper. Particular attention is paid to the form of the load-displacement dependencies, and the region and extent of their deviation from those for 'ideal' geometries.

The mathematical treatment commonly used in the context of experimental indentation analysis and interpretation is that of Sneddon [1] by the technique of integral transforms. An alternative approach particularly suited to axisymmetric problems relies on the use of integral complex potential first proposed by Love [2]. The approach was developed by Green and Zerna [3] and Collins [4], applied in the context of determining load-displacement relations for elastic bodies by Shield [5] and summarized in much detail by Barber [6] and Hills et al. [7].

The effect of rounded corners of flat or conical indenters has been recently considered by Ciavarella [8]. However, in that paper the emphasis was placed on considering the stress fields within the substrate and determining the strength of contact. The purpose of the present study is different. Tractable and simple analysis is presented of the effect of shape imperfections of flat, conical and spherical punches on the load-displacement relationships. These relationships represent the sort of information which is readily available to the experimentalist. The present analysis provides a useful basis for estimating the uncertainty of interpretation due to indenter tip shape errors.

\subsection{Fundamentals}

Assume that a semi-infinite elastic medium, characterized by Young's modulus $E$ and Poisson's ratio $v$, occupies the region $z \geqslant 0$, where the cylindrical coordinates $r, z, \theta$ are introduced so that the surface corresponds to the $r \theta$ plane, and the $z$ axis is perpendicular to it. A rigid indenter is a body of rotation, its intersection with the $r z$ plane being described by the function $-z=s(r)$, where $s(0)=0$. The indenter is translated a certain distance in the positive $z$ direction, producing an indentation in the elastic substrate. The relationship between the load on the indenter, the surface displacements and the state of stress within the elastic half-space must be found.

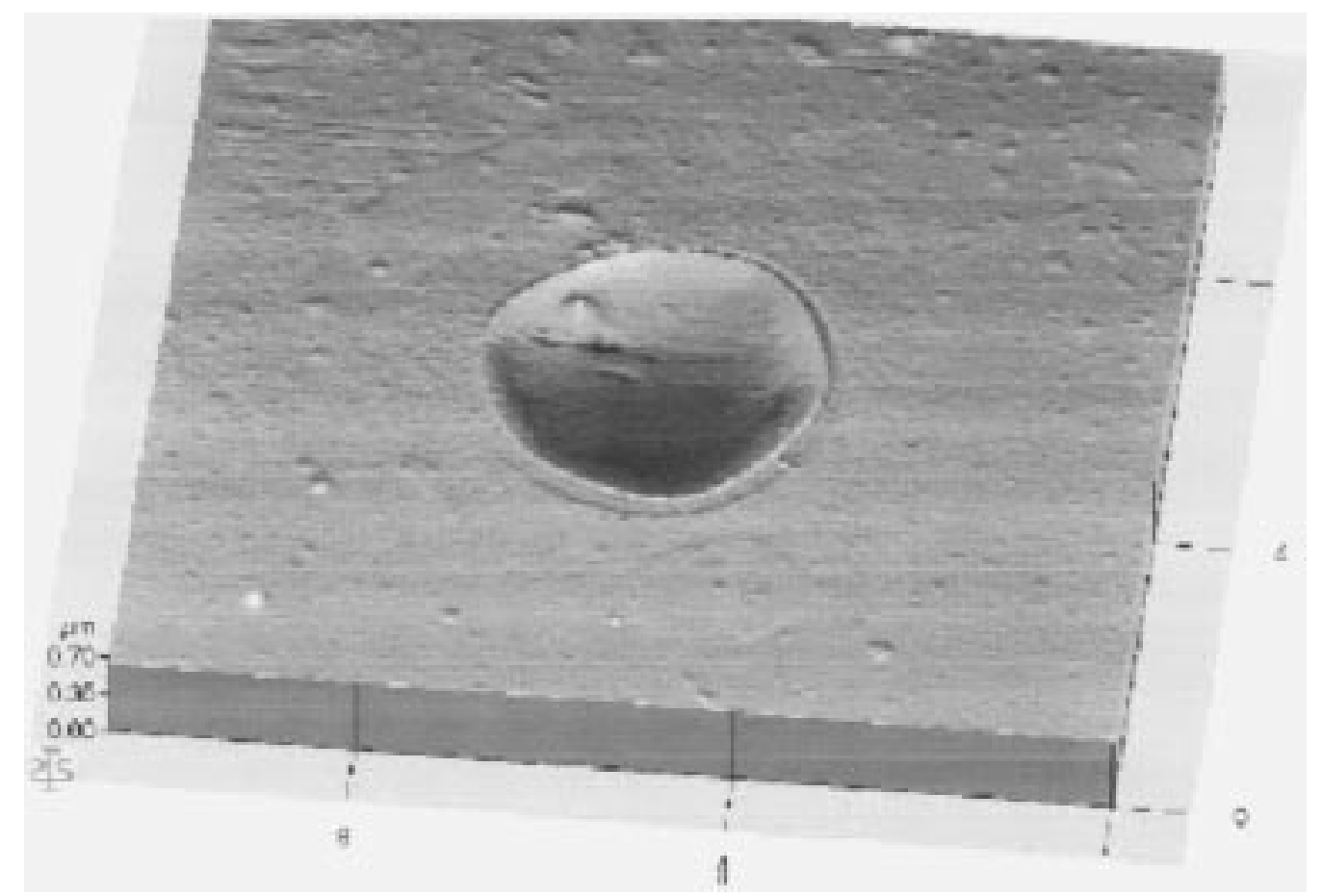

Fig. 1 A surface map of a $500 \mathrm{mN}$ nanoindentation in fused silica with a rounded tip indenter of nominal radius $25 \mu \mathrm{m}$. The image was recorded using an atomic force microscope in contact mode 
In the context of materials indentation testing using continuous recording of load and displacement, the dependence of the solution on the substrate properties $E$ and $v$ and the indenter shape $s(r)$ are of particular interest.

Green and Zerna [3] presented a method of solution suitable for frictionless axisymmetric indentation problems which is based on the ideas of Love [2] and provided a method of solution which avoids the use of integral transforms [1] and Bessel functions, by employing a specially constructed function of complex variable instead.

Boundary conditions for a frictionless axisymmetric indentation problem can be formulated as follows:

$$
\begin{array}{ll}
u_{z}=f(r)=d-s(r), & 0 \leqslant r \leqslant a \\
\sigma_{z z}=\sigma_{y z}=\sigma_{x z}=0, & r>a
\end{array}
$$

where the conditions apply on the plane $z=0, d$ describes the rigid body displacement of the punch, while $a$ denotes the radius of the contact patch, which is generally unknown and must be determined in the course of the solution.

By choosing a special combination of displacement potential functions which provide the solution of general problems of elasticity, the shear stresses at the surface (on the plane $z=0$ ) can be made to vanish identically while, for the axial displacement and stress, it is found that

$$
u_{z}=w(r, z), \quad \sigma_{z z}=\frac{E}{2\left(1-v^{2}\right)} \frac{\partial w}{\partial z}
$$

where $w(r, z)$ denotes an unknown harmonic function $\left(\nabla^{2} w=0\right)$ which must now be found from

$$
\begin{array}{ll}
w(r, 0)=f(r), & 0 \leqslant r \leqslant a \\
\frac{\partial w}{\partial z}(r, 0)=0, & r>a
\end{array}
$$

For a flat punch, the value of contact patch radius $a$ is fixed while, for smooth surface punches (spherical, parabolic, conical, etc.), it must be determined from the condition

$$
\frac{\partial w}{\partial r}(a+0,0)=-s^{\prime}(a+0)
$$

This describes the requirement that the deformed surface and the punch have a common continuous tangent at the edge of the contact area. Equivalently, a requirement may be imposed that the axial stress $\sigma_{z z}$ remains finite at the edge of the contact, $r=a$.

Following Green and Zerna [3], the unknown harmonic potential $w(r, z)$ is sought in the following form:

$$
w(r, z)=\frac{1}{2} \int_{-a}^{a} \frac{g(t) \mathrm{d} t}{\sqrt{r^{2}+(z+\mathrm{i} t)^{2}}} .
$$

Now the task is reduced to determining an unknown function $g(t)$ of only one variable. By considering the asymptotic behaviour of $w(r, z)$ and its derivatives as $z \rightarrow 0$, the following expressions for the contact displacement and stress are obtained:

$$
\begin{aligned}
& \left.u_{z}\right|_{z=0}=\int_{0}^{r} \frac{g(t) \mathrm{d} t}{\sqrt{r^{2}-t^{2}}}=d-s(r), \quad 0 \leqslant r \leqslant a \\
& \left.\sigma_{z z}\right|_{z=0}=\frac{E}{2\left(1-v^{2}\right)} \frac{1}{r} \frac{\partial}{\partial r} \int_{r}^{a} \frac{\operatorname{tg}(t) \mathrm{d} t}{\sqrt{t^{2}-r^{2}}}
\end{aligned}
$$

The problem of finding $g(t)$ is solved by employing the Abel integral transform to invert equation (7) [6]. This leads to

$$
g(t)=\frac{2}{\pi}\left[d-t \int_{0}^{t} \frac{s^{\prime}(r) \mathrm{d} r}{\sqrt{t^{2}-r^{2}}}\right]
$$

In order to complete the analysis, the maximum indentation depth $d$ must now be related to the applied load $P$, given by

$$
\begin{aligned}
P & =-2 \pi \int_{0}^{2} \sigma_{z z}(r, 0) r \mathrm{~d} r \\
& =\frac{\pi E}{1-v^{2}} \int_{0}^{a} g(t) \mathrm{d} t
\end{aligned}
$$

This remarkably simple equation is applicable in the general case of frictionless axisymmetric indentation.

\section{CLASSICAL SOLUTIONS OF THE INDENTATION PROBLEM}

In this section, some well-known results for simple punch shapes are derived within the chosen framework. In particular, the load-displacement dependencies are obtained and will be used in the subsequent analysis as reference.

\subsection{A flat punch}

The solution is perhaps the most straightforward in the case of the flat punch, for which $f(r)=0$ and, from equation (9), $g(t)=2 d / \pi$. The size of the contact patch is determined by the punch shape, and the load-displacement dependence is found to be linear since, from equation (10),

$$
P=\frac{2 E a d}{1-v^{2}}
$$




\subsection{A Hertzian punch}

As an example, consider the case of Hertzian indentation:

$$
f(r)=d-\frac{1}{2} \alpha r^{2}, \quad f^{\prime}(r)=-\alpha r
$$

where, if the surface is indented using a ball of radius $R$, then the punch curvature $\alpha$ is given by $\alpha=1 / R$. From equation (9),

$$
g(t)=\frac{2}{\pi}\left(d-\alpha t^{2}\right)
$$

The requirement of surface smoothness, or pressure finiteness, at the edge of contact may be invoked now. In terms of the function $g(t)$ it translates into the condition $g(a)=0$, which gives the expression for the maximum indentation depth $d=\alpha a^{2}=a^{2} / R$. Note that at the edge of contact the vertical displacement $u_{z}(a)=f(a)=d / 2$, which is a well-known result first pointed out by Sneddon [1].

The total load on the punch in the Hertzian case is found using equation (10):

$$
P=\frac{4}{3} \frac{E}{1-v^{2}} R^{1 / 2} d^{3 / 2}
$$

which corresponds to the famous Hertzian solution in the case of rigid punch.

\subsection{A sharp conical indenter}

In this case assume that the punch shape is described by $f(r)=c r$. According to equation (9),

$$
g(t)=\frac{2 d}{\pi}-c t
$$

The size of the contact patch is determined by setting $g(a)=0$, so that $a=2 d /(\pi c)$.

The total load is determined from equation (10):

$$
P=\frac{2 E d^{2}}{\pi\left(1-v^{2}\right) c}
$$

and shows the characteristic quadratic dependence on the indentation depth.

\section{SOME SOLUTIONS FOR 'BLUNTED' INDENTERS}

In the context of experimental hardness measurements and indentation studies it is customary to choose one of classical punch shapes to describe the substrate deformation under the contact. The distinction is usually made between sharp and smooth punches, which in the simplest terms is often translated into comparing conical or parabolic indenter shapes.

Because of the stress singularity near the conical indenter tip, the substrate material is likely to undergo yielding, so that an accurate indentation model must take account of plasticity. In the limit of large-scale plastic flow under a sharp indenter, the load-displacement dependence is quadratic $\left(P \propto d^{2}\right)$.

In the case of a smooth punch, on the other hand, elastic contact response may be expected to dominate, at least for a range of low loads and indentation depths. The Hertzian model can then be taken as a reasonable approximation, giving $P \propto d^{3 / 2}$.

However, neither of the above models alone is sufficient for the interpretation of indentation results. No real indenter tip can be made ideally sharp or rounded. Hence, the low-load response is likely to be predominantly elastic, even with nominally sharp indenters, while at higher loads a transition to a different load-displacement regime is likely to occur. The nature of this transition, and the magnitude of errors arising from tip shape inaccuracies, may be investigated by studying the solutions for non-ideal indenter geometries, derived in the following section.

\subsection{A Hertzian punch with a 'flat spot'}

Now a particular smooth punch geometry is addressed, which is of direct relevance to the analysis of nanoindentation unloading curves. Namely, consider an indenter with a 'flat spot' of certain radius $b$ in the middle, with the displacement given by the function

$$
f(r)= \begin{cases}d, & r \leqslant b \\ d-\frac{\alpha(r-b)^{2}}{2}, & b \leqslant r \leqslant a\end{cases}
$$

The unknown function $g(t)$ can be found by employing equation (9) where

$$
f^{\prime}(r)= \begin{cases}0, & r \leqslant b, \\ -\alpha(r-b), & b \leqslant r \leqslant a,\end{cases}
$$

so that

$$
g(t)=\frac{2}{\pi}\left[d+\operatorname{sgn}(t-b) \alpha t \int_{b}^{t} \frac{(r-b) \mathrm{d} r}{\sqrt{t^{2}-r^{2}}}\right]
$$

$$
0 \leqslant t \leqslant a
$$

Integrating this expression, $g(t)$ is found in the form 


$$
\begin{gathered}
g(t)=\frac{2}{\pi}\left\{d+\operatorname{sgn}(t-b) \alpha t\left[\frac{b \pi}{2}-b \arcsin \left(\frac{b}{t}\right)\right.\right. \\
\left.\left.-\sqrt{t^{2}-b^{2}}\right]\right\}
\end{gathered}
$$

Once again, the condition of vanishing pressure at the edge of the contact zone $t=a$ leads to the requirement $g(a)=0$. This allows the indentation depth $d$ to be related to the contact patch radius $a$ as follows:

$$
\begin{aligned}
d & =\alpha\left[a \sqrt{a^{2}-b^{2}}+a b \arcsin \left(\frac{b}{a}\right)-\frac{\pi a b}{2}\right] \\
& =\alpha a^{2} \phi(k)
\end{aligned}
$$

Here $k$ is one of the following parameters characterizing the punch shape:

$$
k^{\prime}=\frac{b}{a}, \quad k=\sqrt{1-k^{\prime 2}}=\frac{\sqrt{a^{2}-b^{2}}}{a}
$$

and

$$
\begin{aligned}
\phi(k) & =\sqrt{1-k^{\prime 2}}+k^{\prime} \arcsin k^{\prime}-\frac{\pi k^{\prime}}{2} \\
& =k-k^{\prime} \arcsin k
\end{aligned}
$$

Invoking equation (10) leads to the expression for the total load given by

$$
P=\frac{E \alpha a^{3}}{1-v^{2}}\left[\phi(k)+\frac{k^{3 / 2}}{3}\right]
$$

The parameter $k$ provides a convenient descriptor of the stage in the indentation process. At the very start (very low loads and contact patch radius equal to $b$ ), $k=0$, and $k$ increases with increasing indentation depths, contact patch radii and applied loads. In the limit of very large depths, $a>b$ and the 'flat spot' can be neglected. That this is indeed the case is confirmed by verifying that Hertzian formulae are recovered for $k=1, \phi(k)=1$.

Consider the relationship between the total load and the indentation depth for a blunted punch shape. Introduce another dimensionless parameter $\beta=\alpha b$. Then, normalizing the penetration depth $d$ with respect to its product by the flat spot radius $\beta b, \delta=d /(\beta b)$, from equation (20),

$$
\delta=\frac{\phi}{k^{\prime 2}}
$$

The contact load may be normalized with respect to the quantity $\beta E b^{2} /\left(1-v^{2}\right)$, giving

$$
p=\frac{P\left(1-v^{2}\right)}{\beta E b^{2}}=\frac{1}{k^{\prime 3}}\left(\phi+\frac{k^{3 / 2}}{3}\right)
$$

The dependence of $p$ on $\delta$ follows from the two above expressions implicitly.

In order to highlight the nature of the load-displacement dependence arising for the punch geometry considered, it is illuminating to analyse the asymptotic behaviour of $p$ and $\delta$ for extreme values of $k$. At very low loads at the start of indentation, $k=\epsilon<1$ is small, while $k^{\prime}=\sqrt{1-\epsilon^{2}} \approx 1$. From equation (22) it follows that

$$
\phi=\epsilon-\sqrt{1-\epsilon^{2}} \arcsin \epsilon \approx \frac{\epsilon^{3}}{3}
$$

and, therefore,

$$
\delta \approx \frac{\epsilon^{3}}{3}, \quad p \approx \frac{\epsilon^{3}+\epsilon^{3}}{3}=\frac{2 \epsilon^{3}}{3}
$$

so that, for small $k$,

$$
p \approx 2 \delta, \quad k<1
$$

This remarkable result demonstrates, by comparison with equation (11), that at shallow depths a blunt Hertzian indenter produces a response similar to that for a flat punch. This finding can be understood by noting that the shallow indentation solution is unaffected by modifying the value of the product $a b$, while progressively improved approximations to the flat punch shape can be obtained within the framework of the present analysis by considering the transition to the limit $a b \rightarrow \infty$.

For the values of $k$ approaching one, on the other hand, $\phi \rightarrow 1$, while $k^{\prime}=\epsilon$ plays the role of a small parameter. It is easily observed that

$$
\delta \approx \frac{1}{\epsilon^{2}}, \quad p \approx \frac{4}{3 \epsilon^{3}}
$$

so that

$$
p \approx \frac{4 \delta^{3 / 2}}{3}, \quad k^{\prime}<1
$$

This behaviour is entirely to be expected; in the limit of deep indentations the influence of the 'flat spot' may be expected to diminish, so that the solution for a Hertzian punch is recovered.

The dependence of the normalized load $p$ on the normalized indentation depth $d$ is shown in Fig. 2 for three cases: a flat punch, a Hertzian punch and a 'blunted' Hertzian punch. While the two classical solutions provide correct descriptions of the asymptotic behaviour of the third, it is important to note that the presence of a 'flat spot' produces a significant variation in the load-displacement 


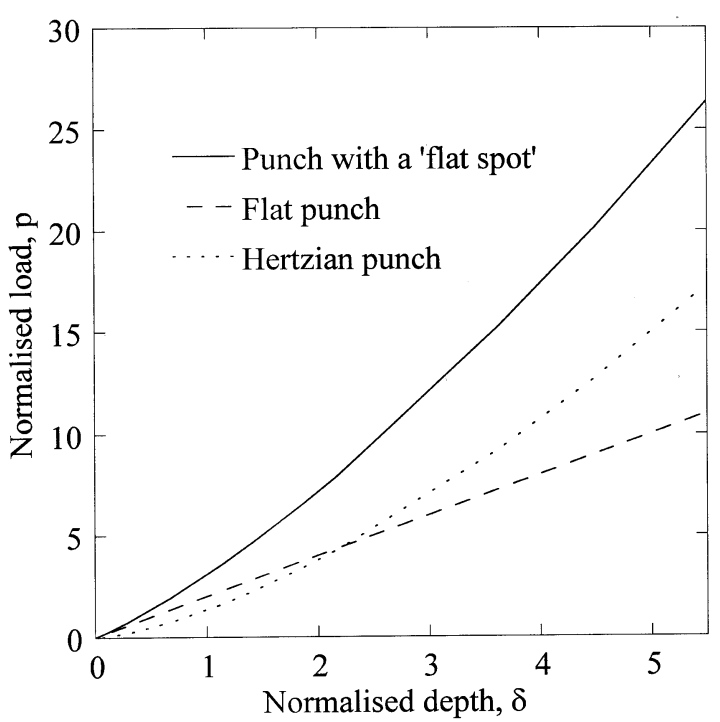

Fig. 2 The dependence of the normalized load $p$ on the normalized indenter displacement $d$ for a blunted Hertzian tip with a 'flat spot', compared with the solutions for an ideal Hertzian indenter and a flat punch

dependence; even at the normalized depth of 5 , the difference between the present solution and that for an ideal Hertzian punch exceeds 25 per cent.

\subsection{A conical punch with a rounded tip}

Now consider indentation by a punch in the shape of a cone, which has a tip blunted to a radius $b$ so that its shape can be approximated (to a first degree) by a parabola (Hertzian case). Clearly, at low applied loads the loaddisplacement response will be directly equivalent to that obtained in an earlier section for a Hertzian indenter. However, once the contact patch radius exceeds that of the blunted tip, a different sort of behaviour will set in. It may be expected that for larger loads the influence of the blunting will diminish. However, it is also obvious that a blunt indenter does not create any stress singularity at the tip, which makes this case fundamentally different from indentation with a sharp tip. It is possible to pose the question, then, of whether in the limit of large loads the solution for a blunted cone approaches that for a sharp cone.

Consider the case when the contact patch radius $a$ is not less than the blunting radius $b$, so that the displacement within the contact zone can be described by

$$
f(r)= \begin{cases}d-\frac{\alpha r^{2}}{2}, & r \leqslant b \\ d-\alpha b\left(r-\frac{b}{2}\right), & b \leqslant r \leqslant a\end{cases}
$$

$$
g(t)=\frac{2}{\pi}\left[d-\alpha t \int_{0}^{t} \frac{[r, b] \mathrm{d} r}{\sqrt{t^{2}-r^{2}}}\right]
$$

where the following notation has been used:

$$
[r, b]= \begin{cases}r, & r \leqslant b \\ b, & r \geqslant b\end{cases}
$$

Integration leads to

$$
g(t)=\frac{2}{\pi}\left\{d-\alpha t^{2}+\alpha t\left[\sqrt{t^{2}-b^{2}}-b \arccos \left(\frac{b}{t}\right)\right]\right\}
$$

The requirement that the pressure falls to zero at the contact edge yields $g(a)=0$, so that the penetration depth

$$
d=\alpha a^{2} \psi(k), \quad \psi(k)=1-k+k^{\prime} \arcsin k
$$

where the parameters $k$ and $k^{\prime}$ were defined in equation (21).

The total contact load $P$ can now be found using equation (10), leading to the expression

$$
P=\frac{2 E \alpha a^{3}}{1-v^{2}}\left\{\psi(k)-\frac{1}{3}+H(a-b)\left[\frac{1-\psi(k)}{2}-\frac{k^{3}}{6}\right]\right\}
$$

In order to derive the load-displacement dependence from the above results, it is important to appreciate that the expressions for the parameters $d$ and $P$ contain the contact patch diameter, which varies with applied load. Once again, the normalized values $\delta$ and $p$ should be used, as defined in the previous section, leading to the following implicit (in terms of $k)$ description of the region $\delta>1(a>b)$ :

$$
\delta=\frac{\psi(k)}{k^{\prime 2}}, \quad p=\frac{2}{k^{\prime 3}}\left[\frac{1-k^{3}}{6}+\frac{\psi(k)}{2}\right]
$$

It is interesting to consider the asymptotic behaviour of the solution at the extreme values of the loading parameter $k$. Firstly, note that, while the rounded tip alone is in contact with the substrate, the Hertzian solution of an earlier section is simply reproduced by setting $a=b, k=0$, $k^{\prime}=1, \psi(k)=1$.

Now consider the case of small $k=\epsilon$; then $k^{\prime} \approx$ $1-\epsilon^{2} / 2$ and $\psi \approx 1-\epsilon^{3} / 3$. Therefore, $d=\alpha a^{2}\left(1-\epsilon^{3} / 3\right)$ and

$$
P \approx \frac{4}{3} \frac{E \alpha a^{3}}{1-v^{2}}\left(1-\frac{\epsilon^{3}}{2}\right)
$$

and 


$$
\delta \approx 1-\frac{\epsilon^{3}}{3}, \quad p \approx \frac{4}{3}\left(1-\frac{\epsilon^{3}}{2}\right), \quad p \approx 2 \delta-\frac{2}{3}
$$

The last relation indicates that, when the expanding contact patch reaches the limit of the rounded region, the initial departure from the Hertzian load-displacement behaviour $\left(p=4 \delta^{3 / 2} / 3\right.$ ) occurs in the second-order term in the series expansion. In other words, the two segments of the loaddisplacement curve, corresponding to $\delta<1$ and $\delta>1$, have a common tangent at the point $\delta=1, p=4 / 3$.

In the limit of large loads, $k \rightarrow 1$, while $k^{\prime}=\epsilon$ becomes small and $\psi \approx \pi \epsilon / 2$. Substitution results in $\delta \approx \pi /(2 \epsilon)$ and $p \approx \pi /\left(2 \epsilon^{2}\right)$, so that finally, in the limit of large loads,

$$
p \approx \frac{2}{\pi} \delta^{2}
$$

This result corresponds to that given for the case of a sharp cone indenter [cf. equation (15)].

An illustration of the load-displacement behaviour for a rounded-tip conical indenter, in comparison with an ideally sharp cone and a Hertzian punch, is given in Fig. 3. Once again, the magnitude of the discrepancy between the three solutions considered here should be noted in particular, which emphasizes the importance of choosing a correct approximation for the indenter shape.

The subject of choosing a procedure for the determination of the best shape approximation is addressed in the following section.

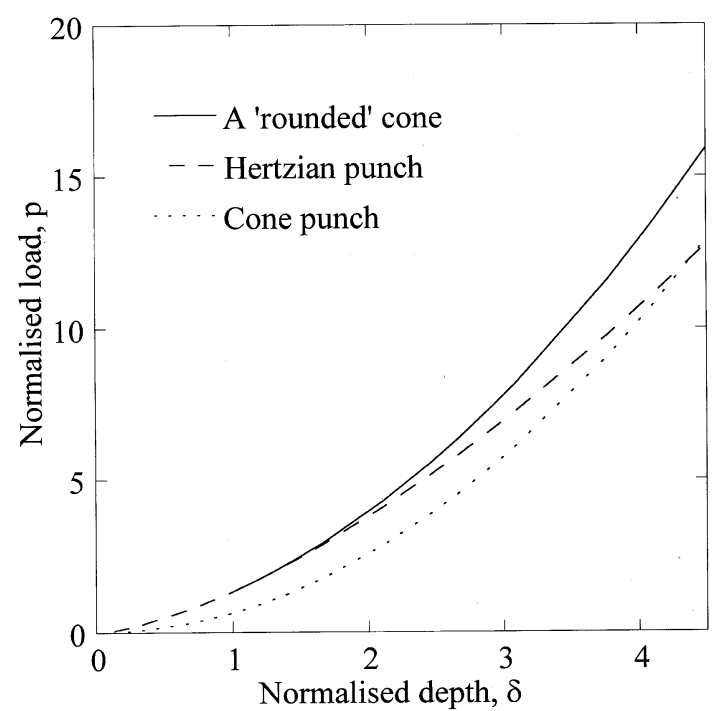

Fig. 3 The dependence of the normalized load $p$ on the normalized indenter displacement $d$ for a rounded cone tip, compared with the solutions for an ideally sharp cone and a Hertzian tip

\section{GENERAL CASE OF PUNCH BLUNTING}

The above results demonstrate that correct interpretation of elastic indentation load-displacement traces in terms of the substrate's elastic properties is impossible without detailed knowledge of the tip shape. The natural extension of the approach presented in the previous section is to approximate the punch shape by a power series, so that

$$
f(r)=d-\sum_{k=1}^{n} b_{k} r^{k}
$$

where the constant term can be omitted from the power series, since it corresponds to the rigid translation of the indenter, accounted for by the indentation depth $d$. It is assumed, as usual, that the inclusion of a moderate number $n$ of terms would provide a sufficiently accurate approximation.

The distribution function $g(t)$ can be determined from equation (9) as follows:

$$
g(t)=\frac{2}{\pi}\left(d-\sum_{k=1}^{n} k b_{k} s_{k-1} t^{k}\right)
$$

where

$$
s_{k}=\int_{0}^{\pi / 2} \sin ^{k} \phi \mathrm{d} \phi
$$

For example, $s_{0}=\pi / 2, \quad s_{1}=1, \quad s_{2}=\pi / 4, \quad s_{3}=2 / 3$, $s_{4}=3 \pi / 16, s_{5}=8 / 15$, etc. Generally, for $k=2 m+1$, $s_{2 m+1}=(2 m) ! ! /(2 m+1) ! ! \quad$ and, for $k=2 m, \quad s_{2 m}=$ $(\pi / 2)(2 m-1) ! ! /(2 m) ! ![9]$.

The condition of smooth contact at the edge of the contact patch $(r=a)$ yields the relationship between $d$ and $a$ in the form

$$
d(a)=\sum_{k=1}^{n} c_{k} a^{k}, \quad c_{k}=k b_{k} s_{k-1}
$$

The total indentation load can also be calculated in terms of the contact patch radius $a$ as follows:

$$
\begin{aligned}
P(a) & =\frac{\pi E}{1-v^{2}} \int_{0}^{a} g(t) \mathrm{d} t \\
& =2 E^{*} \int_{0}^{a}\left(d-\sum_{k=1}^{n} c_{k} t^{k}\right) \mathrm{d} t \\
& =2 E^{*} \sum_{k=1}^{n} \frac{k c_{k} a^{k+1}}{k+1}
\end{aligned}
$$


where $E^{*}=E /\left(1-v^{2}\right)$.

The above equations provide a parametric representation of the load-displacement dependence $P(d)$ observed in an indentation experiment. Some of the classical results discussed earlier can be readily obtained from the present formulation. For example, for a sharp cone punch let only the non-zero coefficient in the power series (36) be $b_{1}=1$. Then $d=(\pi / 2) a$ and $P=(\pi / 2) E^{*} a^{2}$, and hence the expected dependence $P=(2 / \pi) d^{2}$ is recovered.

For a Hertzian punch of spherical tip shape, only the non-zero coefficient in equation (36) is $b_{2}=1 /(2 R)$, where $R$ is the tip radius. Then $d=a^{2} / R$ and $P=(4 / 3) E^{*} a^{3} / R$; hence the Hertzian indentation law $P=(4 / 3) E^{*} R^{1 / 2} d^{3 / 2}$ is obtained.

The conclusion that can be drawn from the analysis of the present section is that, for any given approximation to the punch shape given by equation (36), a model loaddisplacement dependence can be derived in parametric form. This opens up the possibility of performing punch shape calibration by finding the best match between the model prediction and the experimentally recorded traces, e.g. in terms of some least-squares norm. The relevant analysis is described in the next section.

\section{PUNCH SHAPE CALIBRATION}

Evaluation of the punch shape from known load-displacement dependence is an example of inverse problem in the mechanics of indentation. It is somewhat less common than the alternative formulation, where the punch shape is assumed known, and the mechanical properties of the substrate are being determined. However, it should be clear from the foregoing analysis that punch shape variation has a pronounced effect on the load-displacement dependence and hence must be identified to good precision, if any trust is to be put into subsequent data interpretation.

From a practical viewpoint, before any further analysis is undertaken, it would be reassuring to know that indentation experiments described as 'purely elastic' can be carried out. Such experiments show very good coincidence, to within a fraction of the experimental error, between the loading and unloading traces. Measurements of this kind have indeed been reported in the literature $[\mathbf{1 0}]$ and can be obtained particularly readily for hard ceramic substrates.

For the purposes of numerical manipulation, it is convenient to introduce a normalized parameter $x=a / \bar{a}$, where $\bar{a}$ denotes the contact radius at maximum load. The parametric relationship between $P$ and $d$ can be given by

$$
d(x)=\bar{d} \sum_{k=1}^{n} \bar{c}_{k} x^{k}, \quad P(x)=2 E^{*} \bar{a} \bar{d} \sum_{k=1}^{n} \frac{k \bar{c}_{k} x^{k+1}}{k+1}
$$

where $\bar{c}_{k}=c_{k} \bar{a}^{k} / \bar{d}$.
Note from the above expressions that the maximum contact radius $\bar{a}$ appears in the expression for indentation load only in combination with the substrate plane strain elastic modulus $E^{*}$, so that neither can be evaluated independently. In practice, it is likely that tip calibration would be performed using well-characterized substrates, so that $E^{*}$ can be assumed known. Then, once $\bar{c}_{k}$ are determined, the maximum contact radius $\bar{a}$ and the punch shape function (36) are calculated from

$$
\bar{a}=\frac{\bar{P}}{2 E^{*} \bar{d} \sum_{k=1}^{n} k \bar{c}_{k} /(k+1)}, \quad b_{k}=\frac{\bar{d} \bar{c}_{k}}{k s_{k} \bar{a}^{k}}
$$

where $\bar{P}$ denotes the maximum load.

Now the strategy that can be used in order to determine the unknown model parameters $\bar{c}_{k}$ is elaborated. The mismatch between the measured $\hat{P}(d)$ and modelled $P(d)$ dependencies is described by selecting a number $M$ of 'collocation points' at which the differences will be computed, squared and added up. The error, or penalty functional, is denoted by $J$ :

$$
J(\overline{\boldsymbol{c}})=\sum_{m=1}^{M}\left[\hat{P}\left(d_{m}\right)-P\left(d_{m}\right)\right]^{2}
$$

In the above, the sum-of-squares error $J$ is considered as a function of the vector $\overline{\boldsymbol{c}}$ of power series coefficients $\bar{c}_{k}$. Minimization of $J$ with respect to $\bar{c}_{k}$ provides a leastsquares fit of the model to data through the points $d_{m}$, $m=1, \ldots, M$.

In most cases the experimental traces show infinitesimal contact stiffness at the start of indentation, i.e. zero derivative $\mathrm{d} P / \mathrm{d} d$ at $d=0$. In such cases, the coefficient $\bar{c}_{1}$ may be assumed to vanish. This is a reasonable assumption, since no real indenter is ideally sharp, and hence for small radii the tip shape is always approximately parabolic.

It is important to note that the first of equations (41) contains some implicit constraints on the model parameters. The first of these is a linear constraint which can be expressed as follows:

$$
\sum_{k=1}^{n} \bar{c}_{k}=1
$$

In practice this requirement may be enforced as follows. It is assumed that the series coefficients sum to unity, thus reducing the number of free modelling parameters by one. The coefficient $\bar{c}_{n}$ is calculated using

$$
\bar{c}_{n}=1-\sum_{k=2}^{n-1} \bar{c}_{k}
$$

Another constraint is associated with the requirement that 
in the model neither the indentation depth $d$ nor the load $P$ may be allowed to become negative.

The proposed procedure was applied to low load indentations in fused silica. Load-displacement traces showed complete elastic recovery upon unloading and very good repeatability between indents performed at different locations. A single trace was obtained by averaging the results of ten indentations. The load-displacement function obtained in this way was then compared with the predictions for a cone indenter and a Hertzian tip (see Fig. 5), demonstrating that neither approach led to adequate agreement.

The tip shape calibration procedure was then employed, with the shape function being approximated by the weighted sum of three terms $r^{2}, r^{3}$ and $r^{4}$. The coefficients $c_{n}$ were used as the optimization parameters.

Figure 4 shows a three-dimensional surface plot of the error function (43) versus the values of $\bar{c}_{2}$ and $\bar{c}_{3}$ (it was assumed that $\bar{c}_{1}=0$ and $\bar{c}_{4}=1-c_{2}-c_{3}$ ) for the indentation experiment illustrated in Fig. 5. Figure 5 gives a comparison between the experimental load-displacement trace, and the predictions from the simple Hertzian model, the solution for an ideally sharp cone indenter and the bestfit power series shape approximation. It is clear that significant improvement in the agreement with experimental data can be achieved by employing the fitting procedure outlined above.

\section{DISCUSSION}

The results presented above demonstrate that continuously recording indentation testing can be used in conjunction with appropriate elastic modelling as a diagnostic tool. The

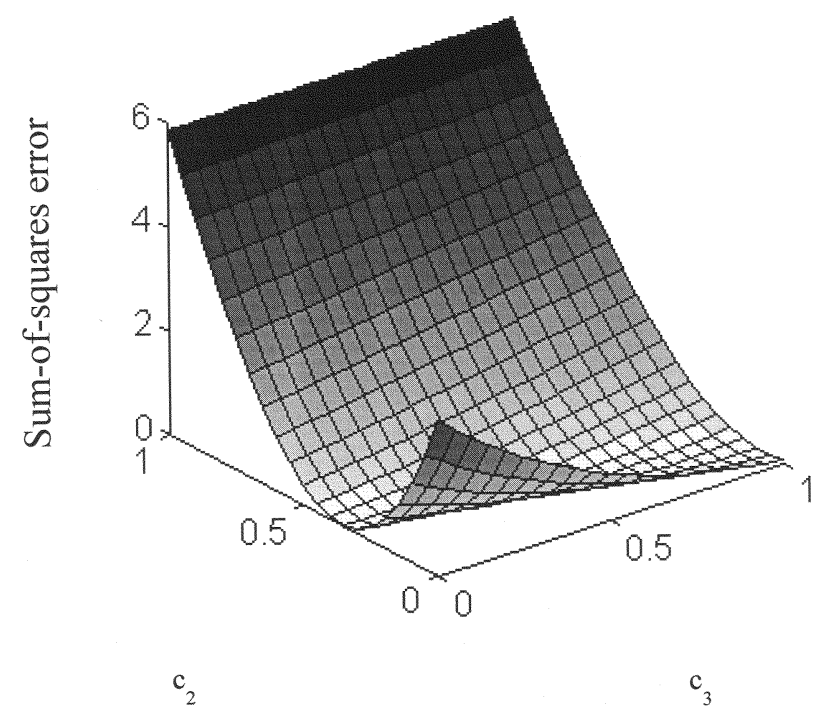

Fig. 4 A surface plot of the sum-of-squares error between experimentally measured and predicted load-displacement traces, against the parameters $c_{2}$ and $c_{3}$

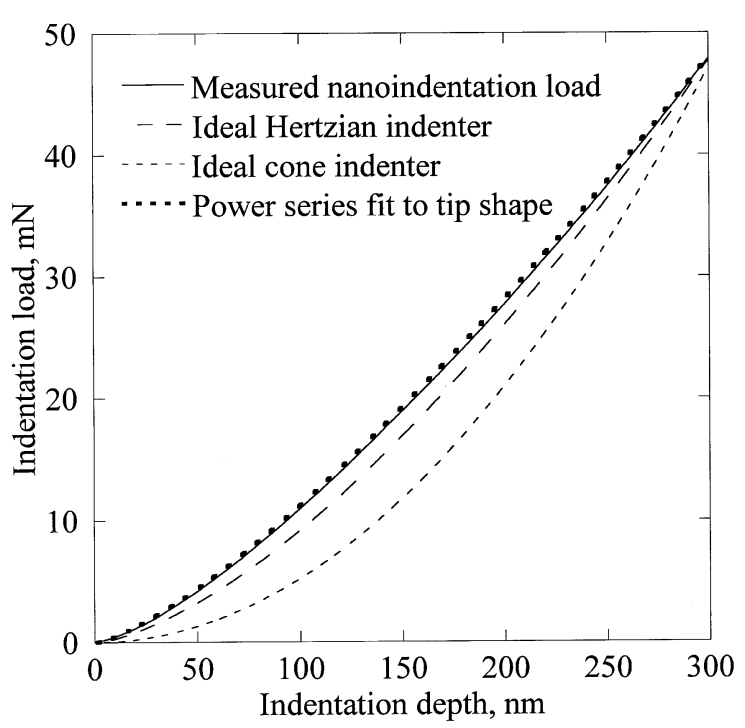

Fig. 5 Comparison between the measured and modelled load-displacement traces for various indenter shapes: sharp cone, ideal Hertzian indenter and power series fit to tip shape, corresponding to the minimum in Fig. 4

method is capable of determining the equivalent axisymmetric punch shape, given precise information about Young's modulus and Poisson's ratio for the substrate. The procedure involves fitting a parametric load-displacement curve to a set of experimental measurements and could be termed elastic punch shape calibration. It should be noted that the conventional tip calibration procedures rely on the substrate plasticity and hence suffer from inherent limitations at very low loads, when tip shape inaccuracies lead to the largest errors.

Once the indenter tip geometry has been found, the same tip can be employed in the analysis of other materials. For example, a similar fitting approach can be used, whereby the substrate's elastic properties are chosen as the optimization parameters.

\section{REFERENCES}

1 Sneddon, I. N. Crack Problems in the Mathematical Theory of Elasticity, 1961 (North Carolina State College, Raleigh, North Carolina).

2 Love, A. E. H. A Treatise on the Mathematical Theory of Elasticity, 1944 (Dover Publications, New York).

3 Green, A. E. and Zerna, W. Theoretical Elasticity, 1954 (Clarendon, Oxford).

4 Collins, W. D. On the potentials of electrified disc. Proc. Lond. Math. Soc., Ser. 3, 1960, 10, 428-460.

5 Shield, R. T. Load-displacement relations for elastic bodies. Z. Angew. Mathematik Physik, 1967, 18, 682-693.

6 Barber, J. R. The solution of elasticity problems for the halfspace by the method of Green and Collins. Appl. Scient. Res., 1983, 40, 135-157.

7 Hills, D. A., Nowell, D. and Sackfield, A. Mechanics of Elastic Contacts, 1993 (Butterworth-Heinemann, Oxford). 
8 Ciavarella, M. Indentation by nominally flat or conical indenters with rounded corners. Int. J. Solids Structs, 1999, 36, 4149-4181.

9 Gradshteyn, I. S. and Ryzhik, I. M. Table of Integrals, Series and Products, 1965 (Academic Press, New York).

10 Hainsworth, S. V., Chandler, H. W. and Page, T. F. Analysis of nanoindentation load-displacement loading curves. $J$. Mater. Res., 1996, 11(8), 1987-1995. 